\title{
Frameless Single Robotic Radiosurgery for Pulmonary Metastases in Colorectal Cancer Patients
}

Jobst C. Von Einem ${ }^{1}$, Sebastian Stintzing ${ }^{2}$, Dominik P. Modest ${ }^{2}$, Michael Wiedemann ${ }^{3}$, Christoph Fürweger ${ }^{4,5}$, Alexander Muacevic 6

1. Hematology, Oncology, and Tumor Immunology (Campus Charité Mitte), Charité - Universitätsmedizin Berlin, Berlin, DEU 2. Oncology, Charité - Universitätsmedizin Berlin, Berlin, DEU 3. Radiation Oncology, European CyberKnife Center, Munich, DEU 4. Medical Physics, European CyberKnife Center, Munich, DEU 5. Stereotaxy and Neurosurgery, University Hospital, Cologne, DEU 6. Radiosurgery, European CyberKnife Center, Munich, DEU

Corresponding author: Jobst C. Von Einem, jobst.einem@gmail.com

\section{Abstract}

\section{Background}

Surgical intervention and radiation therapy are common approaches for pulmonary metastasectomy. The role of minimally invasive techniques in pulmonary metastases remains unclear. Frameless single robotic radiosurgery [CyberKnife (CK); Accuray Incorporated, Sunnyvale, CA] of pulmonary metastases in colorectal cancer (CRC) patients offers high precision local radiation therapy.

\section{Methods}

We analyzed the efficacy and safety of CK treatment for lung metastases in CRC in 34 patients and a total of 45 lesions. The primary endpoint was local control (LC); secondary endpoints were progression-free survival (PFS), overall survival (OS), distant control (DC), and safety-relevant events.

\section{Results}

Of the treated lesions, 34/45 (77.8\%) decreased in size or remained unchanged [complete response (CR), partial response (PR), stable disease (SD)]; 8/45 (17.8\%) lesions increased in size [progressive disease (PD)] and 2/45 (4.4\%) lesions were not evaluable. Local progression was shown in 2 lesions (4.4\%). The median PFS period was six months. In a median follow-up time of 19.4 months, medium OS was 19.9 months (range: 3-61 months). Distant recurrence was observed in 21/34 patients (61.8\%). Intrapulmonary progression occurred in six patients. In $4 / 45$ cases, fiducial placement led to a pneumothorax; three out of four patients needed chest tube insertion. No radiation-associated side effects were reported in $57.8 \%$ of patients. In $10 / 45$ cases $(22.2 \%)$, patients suffered asymptomatic radiographic changes; $7 / 45$ cases (15.6\%) reported a late onset of radiation-associated side effects. Maximal radiation-associated side effects reached the Radiation Therapy Oncology Group/European Organization for Research and Treatment of Cancer

Received 02/06/2020 Review began 02/20/2020 Review ended 03/16/2020 Published 03/18/2020

() Copyright 2020

Von Einem et al. This is an open access article distributed under the terms of the Creative Commons Attribution License CC-BY 4.0., which permits unrestricted use, distribution, and reproduction in any medium, provided the original author and source are credited. (RTOG/EORTC) Grade 1.

\section{Conclusion}

CK treatment of pulmonary metastases is safe and well-tolerated. For metastatic colorectal cancer (mCRC) patients with pulmonary metastases and not eligible for surgery, CK radiation offers a valuable treatment option.

Categories: Radiation Oncology, Gastroenterology, Oncology

Keywords: colorectal cancer, pulmonary metastases, radiation, radiosurgery, cyberknife

\section{Introduction}

About 50-60\% of all patients with colorectal cancer (CRC) develop metastatic disease [metastatic colorectal cancer (mCRC)] [1]. The liver and lung are the most frequent metastases sites of CRC [2]. Approximately 20\% of all patients suffer from pulmonary metastases [3,4]. In 8-10\% of CRC patients, pulmonary metastases occur after previous liver metastases [5]. The development of novel therapeutic regimens, agents, and combinations have led to higher response rates and longer overall survival [6-8]. After complete pulmonary metastasectomy, 5-year survival rates of up to $40 \%$ after resection have been reported $[9,10]$. Since not all patients with pulmonary metastases are eligible for surgical treatment and since techniques are heterogeneous, new treatment options are needed $[1,5,11]$.

Surgery is the gold standard of care for metastasectomy. Some well-established methods in the treatment of liver metastases have been summarized as a "toolbox" to alternatively treat liver metastases if surgery is technically not possible or refused by the patient [12]. For lung metastases, these "tools" are limited or 
questionable with regard to efficacy and toxicity; for instance, radiofrequency ablation (RFA) of peripheral pulmonary tumors have shown heterogeneous results and side-effects [13,14].

It has been reported that non-invasive stereotactic body radiotherapy (SBRT) can reach 2-year local control (LC) rates of approximately $90 \%$ in patients electing not to undergo surgery [14]. This technique usually uses high, hypofractionated dosing and treats small volumes with tight margins [14]. The development of precise tracking options, such as fiducial placement, spine- or lung-tracking, allows the application of eradicating doses to pulmonary metastases and simultaneously spares the healthy lung tissue $[15,16]$.

Encouraged by several reports on radiosurgery treatment in liver metastases and promising reports on radiosurgery treatment in lung metastases, we retrospectively analyzed 34 patients with a total of 45 lesions receiving single-session frameless robotic radiosurgery for CRC with lung metastases $[1,17,18,19]$.

\section{Materials And Methods \\ Study design}

The conducted investigation is a retrospective analysis of the efficacy and safety of single-session robotic radiotherapy of pulmonary metastases in patients suffering from mCRC. The primary endpoint was LC; secondary endpoints were progression-free survival (PFS), overall survival (OS), and distant control (DC).

\section{Patients}

Patients who underwent the procedure from June 2008 to April 2013 were included in our study. Prior to CyberKnife (CK; Accuray Incorporated, Sunnyvale, CA) treatment, patients were reviewed by the multidisciplinary thoracic tumor board of the University Hospital Munich, Grosshadern. Patients suffered from surgically non-resectable pulmonary metastases of CRC. Some patients were also suffering from nonpulmonary metastases as well. Furthermore, patients not willing to undergo surgery in the first place were treated. Pre-treatment included chemotherapy in accordance with the local guidelines, radiotherapy, and surgical metastasectomy. The maximum diameter of metastases had to be less than $5 \mathrm{~cm}$, which was to be determined by a CT scan before the treatment session. Additionally, the total irradiated volume was not to exceed $95 \mathrm{ccm}$. Patients had a lung function test before the intervention. Prior to the first treatment, informed consent to data evaluation was given by all patients in accordance with the guidelines of the local research ethics committee.

\section{Evaluation of response to treatment}

To evaluate the response of irradiated pulmonary metastases, the patients underwent CT scans in an interval of 8-12 weeks. Radiological response evaluation criteria in solid tumors (RECIST) guidelines were used. As differentiation between tumor re-growth and radiogenic inflammation in the RECIST were difficult to apply, short-time follow-up CT scans were performed if clinically indicated. LC was defined as tumor shrinkage or no tumor progress as evaluated in thoracic CT scans. An increase of the tumor volume over $25 \%$ compared to the pre-treatment dimension was defined as local recurrence. Besides, distant recurrence was defined as recurrence in the opposing pulmonary or extra-pulmonary sides.

\section{Evaluation of toxicity}

Side effects were classified as radiation-associated and not-radiation-associated. Pulmonary toxicity was evaluated using pulmonary function tests or CT scans. Furthermore, all patients underwent follow-up evaluations focusing on typical pulmonary symptoms. Morbidity due to marker placement (bleeding, pain) and morbidity due to radiation treatment were evaluated separately. Side effects due to radiation were graded according to the toxicity criteria of the Radiation Therapy Oncology Group (RTOG) and the European Organization for Research and Treatment of Cancer (EORTC). All other safety-relevant events were documented and evaluated.

\section{Marker placement and tracking}

Most patients underwent CT-guided percutaneous placement of one or two cylindrical gold fiducials (AB Medica, Milan, Italy), 5- $\mathrm{mm}$ long and $0.5 \mathrm{~mm}$ in diameter, directly into the metastases prior to radiation. This was done under local anesthesia. Patients had to sign an informed consent, which included warnings of following possible complications: pneumothorax, pulmonary hemorrhage, inadvertent placement of fiducial markers in the pleural space, fiducial marker migration, and unsatisfactory fiducial marker position for use during stereotactic radiotherapy. For patients refusing marker placement or for those not found eligible for marker placement, tracking was done by spine- or lung-tracking.

\section{Radiation}

The 3D target volume was identified on non-contrast-enhanced CT scans. The dose distribution was calculated using the Monte Carlo dose calculation. To cover microscopic tumor spread, a safety margin of 6 $\mathrm{mm}$ was added to the tumor diameter in all three dimensions. All lesions were treated with single-session 
radiosurgery to a maximum dose of $26 \mathrm{~Gy}$. The respiratory motion of the lesion was tracked continuously by a 6-MV compact linear accelerator (LINAC), which was mounted on a six-axis robotic manipulator (CK). Based on the correlation between infrared markers on the patient's chest tracked continuously with external cameras and the position of fiducials detected by two orthogonally positioned X-ray detectors, the position of the linear accelerator was corrected in real time. The radiation beam was directed from a multitude of angles around the patient. After this procedure, which lasted 45 minutes on average, patients were discharged from the institute immediately.

\section{Statistics}

Statistical analysis was done using STATA 10.1 for Macintosh (StataCorp, College Station, TX), IBM SPSS Statistics version 21 (IBM, Armonk, NY), and SAS 9.2 (SAS Institute Inc., Cary, NC).

\section{Results}

There was a total of 45 metastases in 34 CRC patients [male 25 (55.6\%), female 20 (44.4\%)]. The median age was 65 years (range: 41-77 years). Reasons for surgical non-resectability were heterogeneous and included a high number of concomitant diagnoses, prior surgery, or difficult localization of the metastases. The patient characteristics are detailed in Table 1.

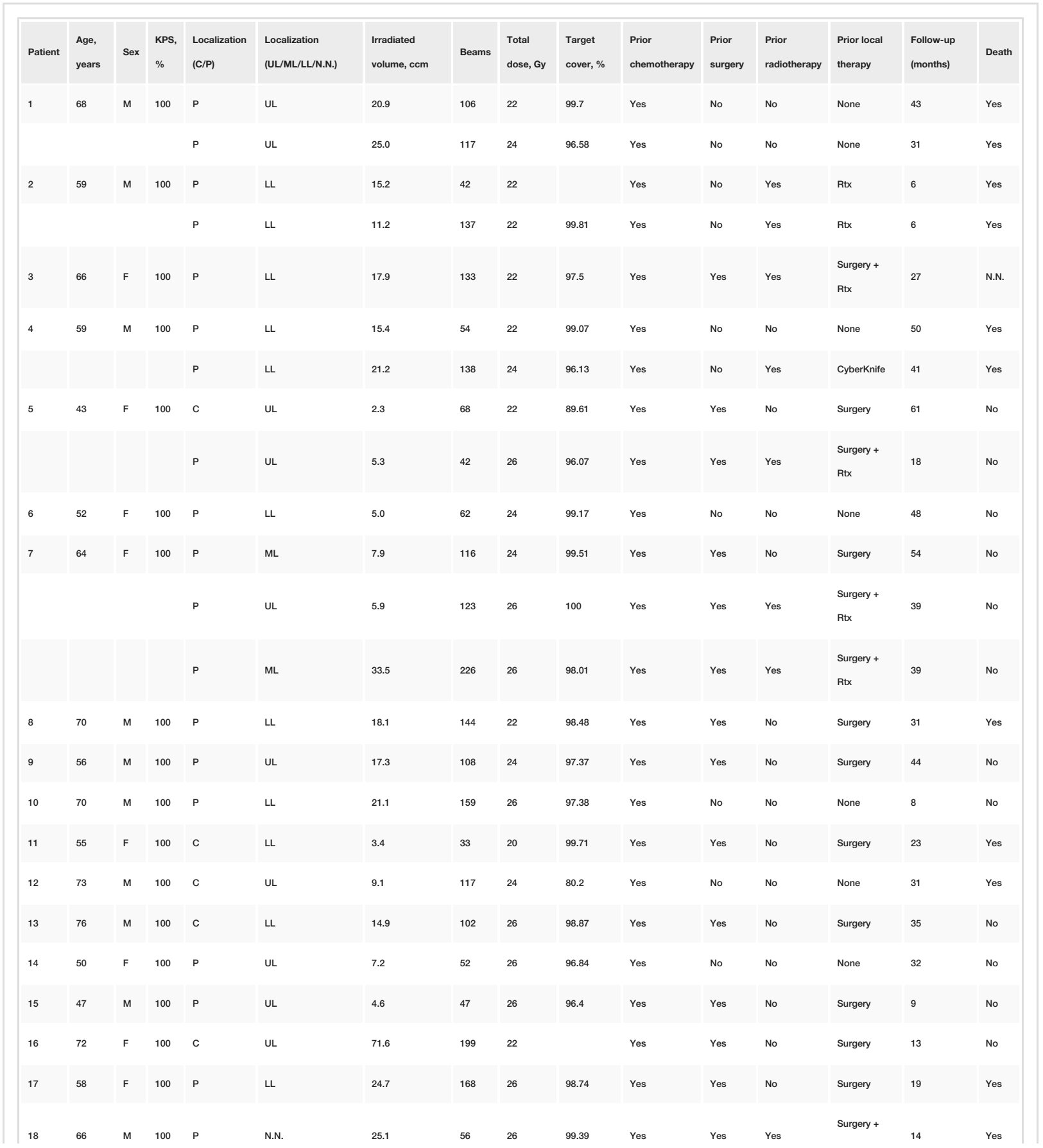




\section{Cureus}

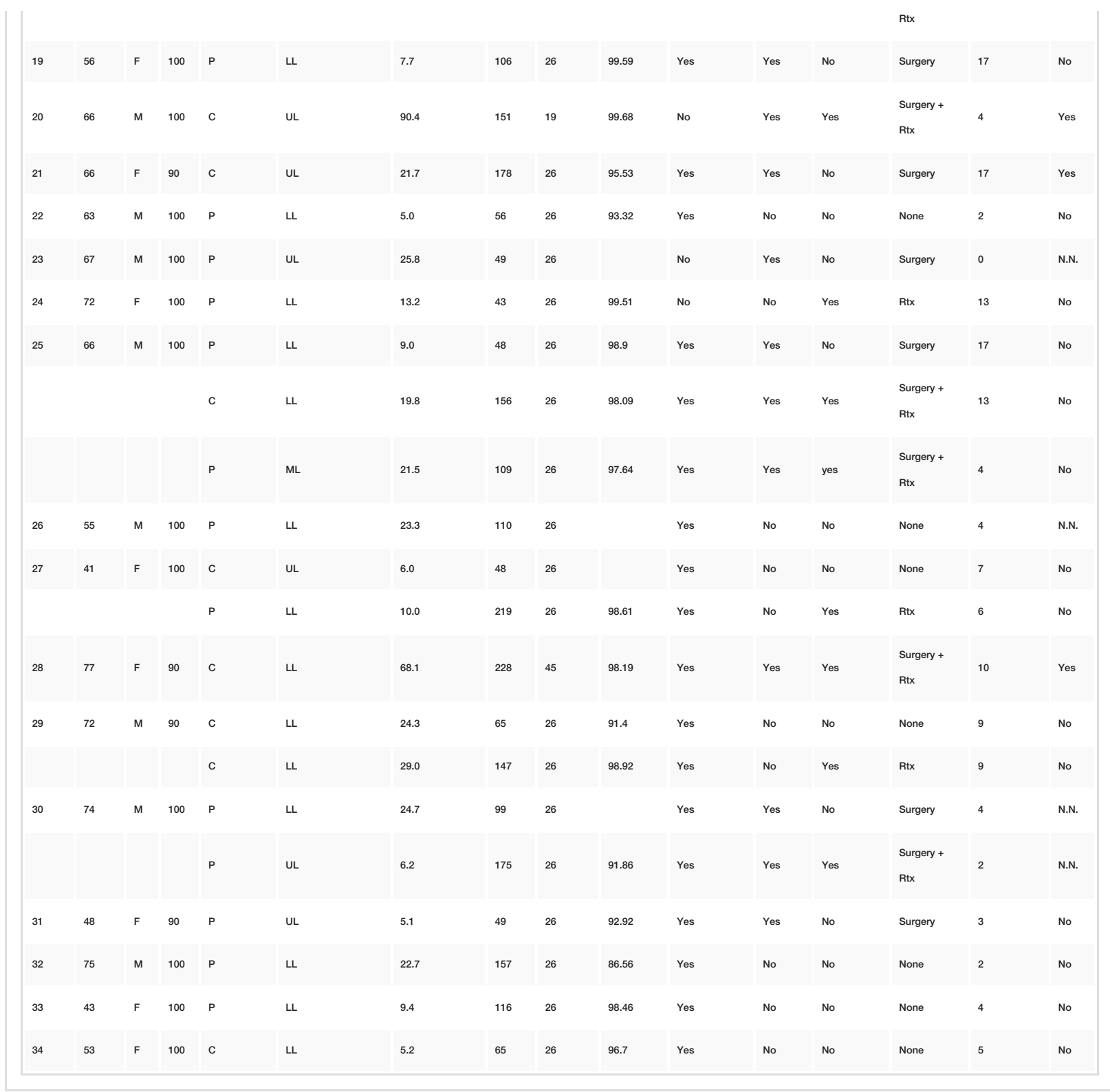

\section{TABLE 1: Patients characteristics}

C: central; P: peripheral; KPS: Karnofsky Performance Status; UL: upper lobe; LL: lower lobe; ML: middle lobe; N.N: nomen nescio (not known); Rtx: radiotherapy

In terms of LC, 35/45 treated lesions (77.8\%) decreased in size or remained unchanged [complete response (CR), partial response (PR), stable disease (SD)], $8 / 45$ (17.8\%) lesions increased in size [progressive disease (PD)], and 2/45 (4.4\%) lesions were not evaluable due to missing follow-up scans. Local progression within the previously irradiated lesion was shown in two lesions (4.4\%) (Figure 1). 


\section{Cureus}

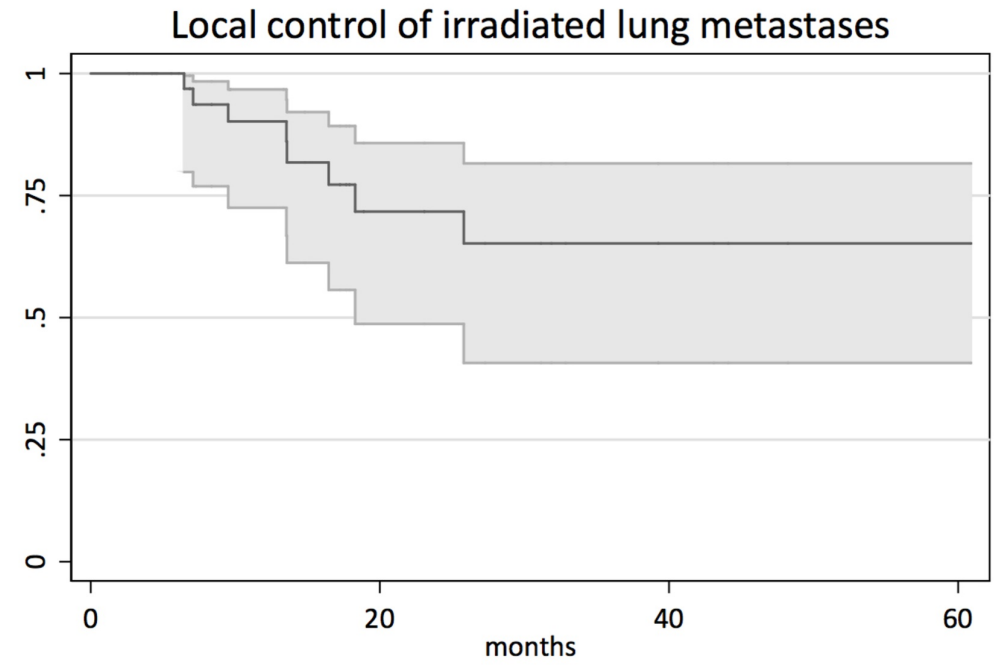

Number at risk

44

12

4

1

$95 \% \mathrm{Cl}-$ Survivor function

FIGURE 1: Kaplan-Meier survival analysis for local control of irradiated lung metastases

$\mathrm{Cl}$ : confidence interval

Median PFS, calculated with the Kaplan-Meier method, was six months. (Figure 2).

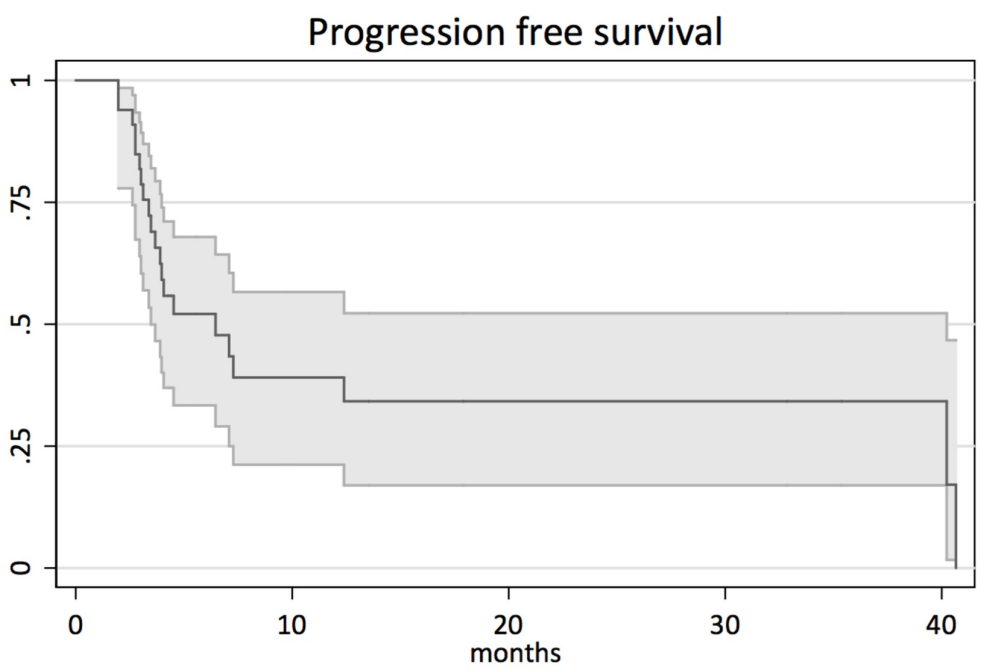

Number at risk

33

\begin{tabular}{|lllc|}
\hline \multicolumn{1}{|c}{8} & 4 & 4 \\
\hline & $95 \% \mathrm{Cl}$ & & Survivor function \\
\cline { 3 - 4 } & & &
\end{tabular}

2

FIGURE 2: Kaplan-Meier survival analysis for progression-free survival Cl: confidence interval

Within a median follow-up time of 19.4 months, medium OS was 19.9 months (range: 3-61 months) (Figure 3). 


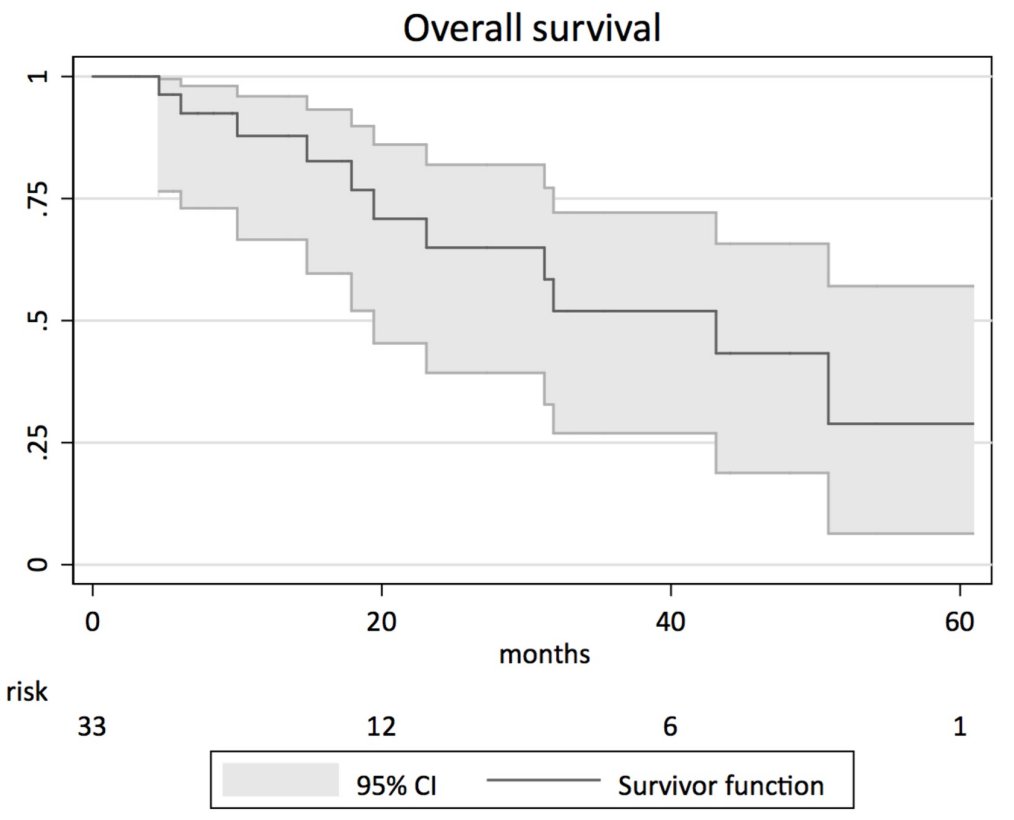

FIGURE 3: Kaplan-Meier survival analysis for overall survival

Cl: confidence interval

Distant recurrence outside the irradiated lung was observed in 21/34 patients, representing $61.8 \%$ of all patients. Intrapulmonary progression in previously non-irradiated sections occurred in six patients.

In 4/45 cases, fiducial placement led to a pneumothorax; three out of these four patients needed chest tube insertion afterward. No radiation-associated side effects were reported in 57.8\% of patients. In 10/45 cases (22.2\%), patients suffered from acute radiation-associated side effects; $7 / 45$ cases (15.6\%) reported a lateonset of radiation-associated side effects. According to the toxicity criteria, RTOG/EORTC maximal radiation-associated side effects reached Grade 1 and were described as asymptomatic pneumonitis.

With a median number of 109.4 beams, a median target cover of $96.7 \%$, and a median isodose of $65.9 \%$, tumors with a mean planning target volume of $18.9 \mathrm{ccm}$ were treated with a median dose of $25.2 \mathrm{~Gy}$.

\section{Discussion}

The approach towards oligometastatic disease in mCRC patients not suitable for or rejecting surgery is challenging, and no standard treatment has been defined so far [12]. The current European Society for Medical Oncology (ESMO) guidelines for the management of metastatic colorectal cancer, therefore, define the local ablative treatment options as "toolbox" [20]. Still, there are notable differences in invasiveness and side effects that require further investigations and ask for the proof of efficacy as compared to surgery, which remains the standard local ablative treatment method. The use of radiation treatment, including SBRT, has shown promising results but data from randomized trials comparing the different local ablative techniques to each other or to surgery is still scarce $[12,21]$.

We report the results of a homogeneously treated group of 34 patients (and a total of 45 lesions) with 1-3 pulmonary metastases due to CRC treated with SBRT using the CK system focusing on safety and efficacy. In terms of safety, we did not determine any radiation-induced toxicity higher than grade 1 pneumonitis (10 patients), defined as asymptomatic radiographic changes. Our findings stand as a testament to the low toxicity and favorable treatment-associated morbidity of single-fraction radiosurgery in patients with pulmonary metastases due to CRC. None of those patients suffered from chronic side effects. In particular, no skin reactions, necrosis, or any other treatment-related toxicities were observed.

Similar treatment was performed by Hof et al. who treated 61 patients with 71 pulmonary metastases of different origins ( $8 / 61$ suffered from pulmonary metastases due to $\mathrm{mCRC}$ ) with stereotactic single-fraction treatment [22]. In this cohort, three patients developed grade 3 toxicity requiring treatment and oxygen for pneumonitis. No grade 4 toxicities occurred. The majority of the patients had grade 1 and 2 toxicities. In their cohort, $70.4 \%$ of all patients developed perifocal changes of the normal lung tissue, detectable by CT scans. Hof et al. displayed no statistically significant correlation between the occurrences of perifocal changes and the tumor volume and the administered radiation dose [22]. But due to the different tumor 
types treated and missing subgroup analysis, the efficacy of SBRT for CRC lung metastases remained unclear.

Another study by Rusthoven et al. included 68 lesions in 38 patients in a multi-institutional phase I/II clinical trial in which they received SBRT in three fractions with doses up to $60 \mathrm{~Gy}$ (9/38 suffering from pulmonary metastases due to $\mathrm{mCRC}$ ). These patients showed no grade 4-5 toxicities. But three patients experienced grade 3 toxicity, including pneumonitis with increased dyspnea, oxygen requirement and a decrease in FeV1, rib fracture after SBRT, and confluent moist desquamation of the skin. Furthermore, four patients developed grade 2 radiation dermatitis, which resolved within three months [18].

The safety of the gold standard procedure, the surgical pulmonary metastasectomy, was investigated in 171 mCRC patients by Meimarakis et al. [3]. Of their 238 surgical interventions ( $n=171$ patients), 156 were performed as wedge resections, 30 as segmental, and 52 as major resections (35 lobectomies, 4 bilobectomies, 1 sleeve bilobectomy, and 12 pneumonectomies). Complications developed in 10 patients. Acute rethoracotomy was performed in one patient with hemorrhagic pleural effusion and empyema. Four patients developed severe pneumonia. Two patients died postoperatively (1.2\%) [3]. In another study, it was shown that peri- and postoperative complications numbers were comparable to the data presented by Meimarakis et al. and included re-intubation, pleural empyema, partial atelectasis, pulmonary embolism, and superficial wound infections [4]. In our cohort, four cases of fiducial placement led to a pneumothorax; three of these four patients needed chest tube insertion. As new techniques such as MR-guided radiotherapy arise, this might be discussed differently; but for now, this approach can be considered safe. In terms of efficacy, an LC rate of 77.8\% (35/45 patients), a median PFS of six months, and a median OS of 19.9 months have been achieved in our study. This is comparable to other non-surgical therapy approaches, but data varies among different local treatment options.

Success in terms of surgery is defined by completeness and margin. LC in resected patients with tumor negative margins is higher and OS, ranging from 25.2 to 51 months, is longer when compared to patients in our study $[3,4,23]$. In these surgery based trials, survival rates differed according to the completeness of pulmonary metastasectomy [3,4]. Meimarakis et al. have stated that the surgical resection of metastases improves survival significantly and coherently that an incomplete resection is associated with poorer outcomes when compared to chemotherapy alone in patients with hepatic and pulmonary colorectal metastases [4]. They showed that complete resection of the pulmonary and hepatic metastases led to a median survival of 66.8 months (31.5 months in patients with incomplete resection) and 30.1 months in patients treated with chemotherapy alone. In their isolated lung metastases cohort, the median survival rate was 35.2 months [3]. 1-, 3-, and 5-year survival rates for patients following R0 resection were 88.8, 52.1, and $32.9 \%$ respectively. Almost identical results were presented by Schuele et al. whose cohort of isolated pulmonary metastases of colorectal primary reached a median survival rate of 33.3 months after surgical metastasectomy [23].

All surgical studies are troubled by the unresolvable bias that holds that only patients with an adequate pulmonary function are suitable for surgical procedures. As an alternative, and for surgically inoperable patients with limited pulmonary metastases, radiation therapy and SBRT in particular may be an option as OS rates for these procedures at 24 months are $33 \%$ and $86 \%$ and LC rates are $53 \%$ to $100 \%$ respectively $[19,21,24-28]$.

A retrospective comparison of outcome after conventional, multi-session SBRT for pulmonary metastasectomy in patients with pulmonary oligometastasis was performed by Widder et al. [26]. In their study, pulmonary metastasectomy was offered as the first-choice treatment and conventional SBRT was offered for patients they considered to be less suitable surgical candidates. More unfavorable prognostic factors were present among the patients treated with conventional SBRT: they were significantly older, had a different distribution of primary tumor origins, and had a shorter metastasis-free interval. In a median follow-up time of 43 months, estimated OS rates at one, three, and five years were $87 \%, 62 \%$, and $41 \%$ for metastasectomy, and $98 \%, 60 \%$, and $49 \%$ for SBRT, respectively. Therefore, and despite the abovementioned selection bias, survival after SBRT was comparable to pulmonary metastasectomy [26].

In terms of technique and in contrast to surgery as well as conventional SBRT, patients in our study presented only once for therapy procedures. In these end-stage tumor patients, time outside a medical institution may be a reasonable marker for the quality of life and thus favors time-efficient procedures.

Our data is limited by several factors. The study was performed retrospectively and at a single center. Even though our cohort of 34 patients (with a total of 45 treated lesions) is comparable to most other studies in this field, the population is still rather small. Furthermore, most of the patients suffered from the oligometastatic disease. Pretreatment conditions, performance status, age, sex, and tumor locations differed partly significantly. Therefore, it is difficult to refer to the OS outcomes of radiosurgery for pulmonary metastases exclusively.

\section{Conclusions}


We can conclude that single-session frameless robotic radiosurgery is a safe, efficient, and convenient method to treat pulmonary oligometastases of CRC in patients not eligible for or rejecting surgery. Radiosurgery offers a treatment option with limited side effects and proficient LC. In accordance with the ESMO guidelines, SBRT using the CK device is an effective method in the "toolbox" to treat mCRC. Further prospective randomized studies are warranted to confirm the effectiveness of radiosurgery and to define the optimal fractionation and dosage.

\section{Additional Information \\ Disclosures}

Human subjects: Consent was obtained by all participants in this study. Ethics committee LMU issued approval 0 . Prior to the first treatment, informed consent to data evaluation was given by all patients in accordance with the guidelines of the local research ethics committee. The name of the local ethics committee is the Ethics committee LMU (Pettenkoferstrasse 8, D-80336 Munich, Germany). Animal subjects: All authors have confirmed that this study did not involve animal subjects or tissue. Conflicts of interest: In compliance with the ICMJE uniform disclosure form, all authors declare the following: Payment/services info: All authors have declared that no financial support was received from any organization for the submitted work. Financial relationships: All authors have declared that they have no financial relationships at present or within the previous three years with any organizations that might have an interest in the submitted work. Other relationships: All authors have declared that there are no other relationships or activities that could appear to have influenced the submitted work.

\section{Acknowledgements}

We thank the patients and their families. We thank Prof. Volker Heinemann for his indefatigable support and advice.

\section{References}

1. Stintzing S, Hoffmann RT, Heinemann V, Kufeld M, Muacevic A: Frameless single-session robotic radiosurgery of liver metastases in colorectal cancer patients. Eur J Cancer. 2010, 46:1026-1032. 10.1016/j.ejca.2010.01.008

2. Neeff H, Horth W, Makowiec F, Fischer E, Imdahl A, Hopt UT, Passlick B: Outcome after resection of hepatic and pulmonary metastases of colorectal cancer. J Gastrointest Surg. 2009, 13:1813-1820. 10.1007/s11605009-0960-1

3. Meimarakis G, Spelsberg F, Angele M, et al.: Resection of pulmonary metastases from colon and rectal cancer: factors to predict survival differ regarding to the origin of the primary tumor. Ann Surg Oncol. 2014, 21:2563-2572. 10.1245/s10434-014-3646-1

4. Meimarakis G, Angele M, Conrad C, et al.: Combined resection of colorectal hepatic-pulmonary metastases shows improved outcome over chemotherapy alone. Langenbecks Arch Surg. 2013, 398:265-76. 10.1007/s00423-012-1046-1

5. Gonzalez M, Robert JH, Halkic N, et al.: Survival after lung metastasectomy in colorectal cancer patients with previously resected liver metastases. World J Surg. 2012, 36:386-391. 10.1007/s00268-011-1381-3

6. Modest DP, Hiddemann W, Heinemann V: Chemotherapy of metastatic colorectal cancer. (Article in German). Internist (Berl). 2014, 55:37-42. 10.1007/s00108-013-3314-8

7. Kopetz S, Chang GJ, Overman MJ, et al.: Improved survival in metastatic colorectal cancer is associated with adoption of hepatic resection and improved chemotherapy. J Clin Oncol. 2009, 27:3677-3683. 10.1200/JCO.2008.20.5278

8. Pastorino U, Buyse M, Friedel G, et al.: Long-term results of lung metastasectomy: prognostic analyses based on 5206 cases. J Thorac Cardiovasc Surg. 1997, 113:37-49. 10.1016/s0022-5223(97)70397-0

9. Fong Y: Surgical therapy of hepatic colorectal metastasis. CA Cancer J Clin. 1999, 49:231-255. 10.3322/canjclin.49.4.231

10. Yedibela S, Klein P, Feuchter K, et al.: Surgical management of pulmonary metastases from colorectal cancer in 153 patients. Ann Surg Oncol. 2006, 13:1538-1544. 10.1245/s10434-006-9100-2

11. Kim HK, Cho JH, Lee HY, Lee J, Kim J: Pulmonary metastasectomy for colorectal cancer: how many nodules, how many times?. World J Gastroenterol. 2014, 20:6133-6145. 10.3748/wig.v20.i20.6133

12. Schmoll HJ, Van Cutsem E, Stein A, et al.: ESMO Consensus Guidelines for management of patients with colon and rectal cancer. a personalized approach to clinical decision making. Ann Oncol. 2012, 23:24792516. 10.1093/annonc/mds236

13. Hoffmann RT, Jakobs TF, Muacevic A, Trumm C, Helmberger TK, Reiser MF: Interventional oncology for lung tumors. (Article in German). Der Radiologe. 2007, 47:1109-1116. 10.1007/s00117-007-1571-Z

14. Siva S, MacManus M, Ball D: Stereotactic radiotherapy for pulmonary oligometastases: a systematic review . J Thorac Oncol. 2010, 5:1091-1099. 10.1097/JTO.0b013e3181de7143

15. Snider JW, Oermann EK, Chen V, et al.: CyberKnife with tumor tracking: an effective treatment for high-risk surgical patients with single peripheral lung metastases. Front Oncol. 2012, 2:63. Accessed: March 17, 2020: https://www.ncbi.nlm.nih.gov/pmc/articles/PMC3386520/. 10.3389/fonc.2012.00063

16. Fumagalli I, Bibault JE, Dewas S, et al.: A single-institution study of stereotactic body radiotherapy for patients with unresectable visceral pulmonary or hepatic oligometastases. Radiat Oncol. 2012, 7:164. Accessed: March 17, 2020: https://www.ncbi.nlm.nih.gov/pubmed/23014094. 10.1186/1748-717X-7-164

17. Stintzing S, Hoffmann RT, Heinemann V, Kufeld M, Rentsch M, Muacevic A: Radiosurgery of liver tumors: value of robotic radiosurgical device to treat liver tumors. Ann Surg Oncol. 2010, 17:2877-2883.

10.1245/s10434-010-1187-9 
18. Rusthoven KE, Kavanagh BD, Cardenes H, et al.: Multi-institutional phase I/II trial of stereotactic body radiation therapy for liver metastases. J Clin Oncol. 2009, 27:1572-1578. 10.1200/JCO.2008.19.6329

19. Rusthoven KE, Kavanagh BD, Burri SH, et al.: Multi-institutional phase I/II trial of stereotactic body radiation therapy for lung metastases. J Clin Oncol. 2009, 27:1579-1584. 10.1200/JCO.2008.19.6386

20. Van Cutsem E, Cervantes A, Adam R, et al.: ESMO Consensus Guidelines for the management of patients with metastatic colorectal cancer. Ann Oncol. 2016, 27:1386-1422. 10.1093/annonc/mdw235

21. Takeda A, Sanuki N, Kunieda E: Role of stereotactic body radiotherapy for oligometastasis from colorectal cancer. World J Gastroenterol. 2014, 20:4220-4229. 10.3748/wjg.v20.i15.4220

22. Hof H, Hoess A, Oetzel D, Debus J, Herfarth K: Stereotactic single-dose radiotherapy of lung metastases . Strahlenther Onkol. 2007, 183:673-678. 10.1007/s00066-007-1724-Z

23. Schüle S, Dittmar Y, Knösel T, Krieg P, Albrecht R, Settmacher U, Altendorf-Hofmann A: Long-term results and prognostic factors after resection of hepatic and pulmonary metastases of colorectal cancer. Int J Colorectal Dis. 2013, 28:537-545. 10.1007/s00384-012-1553-0

24. Wulf J, Haedinger U, Oppitz U, Thiele W, Mueller G, Flentje M: Stereotactic radiotherapy for primary lung cancer and pulmonary metastases: a noninvasive treatment approach in medically inoperable patients. Int J Radiat Oncol Biol Phys. 2004, 60:186-196. 10.1016/j.ijrobp.2004.02.060

25. Takeda A, Kunieda E, Ohashi T, Aoki Y, Koike N, Takeda T: Stereotactic body radiotherapy (SBRT) for oligometastatic lung tumors from colorectal cancer and other primary cancers in comparison with primary lung cancer. Radiother Oncol. 2011, 101:255-259. 10.1016/j.radonc.2011.05.033

26. Widder J, Klinkenberg TJ, Ubbels JF, Wiegman EM, Groen HJ, Langendijk JA: Pulmonary oligometastases: metastasectomy or stereotactic ablative radiotherapy?. Radiother Oncol. 2013, 107:409-413.

10.1016/j.radonc.2013.05.024

27. Kim MS, Yoo SY, Cho CK, et al.: Stereotactic body radiation therapy using three fractions for isolated lung recurrence from colorectal cancer. Oncology. 2009, 76:212-219. 10.1159/000201932

28. Inoue T, Katoh N, Onimaru R, Shirato H: Clinical outcomes of stereotactic body radiotherapy for patients with lung tumors in the state of oligo-recurrence. Pulm Med. 2012, 2012:369820. 10.1155/2012/369820 